\title{
Talcott Parsons y los economistas keynesianos sobre los conceptos de agente individual, organizaciones e instituciones*
}

\author{
Talcott Parsons and the keynesian economists on \\ the concepts of individual agent, organizations and \\ institutions
}

\section{Sagar Hernández-Chuliá}

Profesor del Departamento de Sociología de la Facultad de Ciencias Económicas y Empresariales de la Universidad Autónoma de Madrid, Madrid, España

sagar.hernandez@uam.es

https://orcid.org/0000-0001-8305-0158

Recibido: 07-12-18

Aprobado: $27-08-19$

* Este artículo constituye una adaptación parcial del epígrafe 3.2 "Relación del Keynesianismo con los compiladores en Sociología" de mi Tesis Doctoral "Principales puntos de convergencia entre las escuelas hegemónicas de teoría económica y sociológica desde la Ilustración escocesa hasta nuestros días". El lector puede aproximarse a él de dos maneras. Por una parte, dado que su línea argumental resulta plenamente autocontenida, puede comprenderlo como un texto autónomo que da cuenta de la fundamental convergencia teórica que se produce entre el pensamiento económico keynesiano y el estructural-funcionalismo sociológico de Talcott Parsons en tres categorías específicas: agente individual, organizaciones e instituciones. Por otra, puede considerarlo como una investigación complementaria a un trabajo previo publicado por el mismo autor (Hernández, 2016).

1 Doctor en Sociología. 


\title{
Resumen
}

Con esta investigación, pretendemos aplicar el método comparativo a las contribuciones teóricas que, en torno a los conceptos de agente individual, organizaciones e instituciones, llevan a cabo los principales economistas keynesianos y Talcott Parsons. A la vista de los resultados, concluimos que, si bien Parsons comparte la caracterización de los agentes individuales que llevan a cabo tanto Keynes como los autores postkeynesianos, también se vale de modelos formales, como lo hacen los neokeynesianos. Además, en el área interorganizativa, legitima el papel que puede jugar el Estado en la economía y destaca, como los postkeynesianos, el positivo papel que pueden desarrollar los sindicatos. Finalmente, Parsons también coincide con los economistas postkeynesianos, y muy particularmente con Kaldor, por cuanto comprende las instituciones como fenómenos generales y naturales que pueden presentar un bajo perfil de conflictividad.

Palabras clave: Teoría económica; Sociología; Individuo; Organización; Institución.

JEL: A12, B13, E12, No1, Y80

\begin{abstract}
With this research, we intend to apply the comparative method to the theoretical contributions that, around the concepts of individual agent, organizations and institutions, are carried out both by the main keynesian economists and by Talcott Parsons. In view of the results, we conclude that, although Parsons shares the characterization of the individual agents developed by Keynes and the post-keynesians authors, he also uses formal models, such as the new-keynesians do. In addition, in the inter-organizational area, he legitimizes the role that the State can play in the economy, and highlights, like the post-keynesians, the positive role that unions can develop. Finally, Parsons also coincides with post-keynesian economists, and especially with Kaldor, when he understands institutions as general and natural phenomena that may present a low degree of conflict.
\end{abstract}

Keywords: Economic theory; Sociology; Individual; Organization; Institution.

¿Cómo citar este artículo? / How to quote this article?

Hernández-Chuliá, S. (2020). Talcott Parsons y los economistas keynesianos. Sobre los conceptos de agente individual, organizaciones e instituciones. Sociedad y economía, (39), 113-133 https://doi.org/10.25100/sye.voi39.8565 


\section{Introducción}

Antes de referirnos a los puntos de confluencia entre el pensamiento económico keynesiano y el estructural-funcionalismo sociológico de Talcott Parsons, debemos preguntarnos a qué nos referimos exactamente cuándo nos valemos del término "keynesianismo". Lo primero que debemos señalar, a este respecto, es que lejos de tratarse de una doctrina económica uniforme podemos identificar, al menos, dos corrientes claramente diferenciadas. Así, por una parte, nos encontramos con la obra del fundador de la escuela y sus seguidores más cercanos, los postkeynesianos; y, por otra, con las aportaciones de los economistas neokeynesianos.

La relación que mantiene Keynes con los autores postkeynesianos se caracteriza por una gran proximidad, tanto en lo doctrinario como en lo personal. En lo doctrinario, las propuestas de tal grupo de economistas coinciden, tanto con la "economía de Keynes" (Leijonhufvud, 1968) como con su pretensión de superar las aportaciones teóricas neoclásicas. En lo personal, además de ser colegas suyos en la Universidad de Cambridge, mantienen frecuentes reuniones con él en el Circus. Este es un círculo de investigadores dedicado a analizar semanalmente las aportaciones que Keynes realiza a la teoría económica en su Tratado Sobre el Dinero (Keynes, 1930). Entre sus miembros más destacados encontramos autores de la talla de Joan Robinson, Richard Kahn, Piero Sraffa o Nicholas Kaldor. El animado debate intelectual que tiene lugar en el Circus favorece, por ejemplo, que Keynes reconozca la influencia que ha ejercido sobre él la lectura de la Economía de la Competencia Imperfecta de Robinson (1933) a la hora de escribir su Teoría General (Keynes, 1936), o que tome de Kahn su concepto de multiplicador y el uso de la curva marshalliana de oferta a corto plazo.

Por su lado, la doctrina de los economistas neokeynesianos, que no mantienen una relación directa con el fundador de la escuela, puede ser caracterizada, como hace Leijonhufvud (1968), como "economía keynesiana". Dicha aproximación remite a las investigaciones realizadas por autores de la talla de Paul A. Samuelson o John Hicks y se caracteriza por la incorporación de postulados teóricos procedentes de la escuela neoclásica. Así, por ejemplo, Samuelson (1970) denomina su propio programa de investigación "síntesis neoclásica", y lo define como el resultado de la confluencia teórica del análisis de determinación de la renta, propio de la macroeconomía de Keynes, con los principios microeconómicos neoclásicos sobre la formación de precios elaborados por Walras, Pareto, Menger y Marshall.

Por otra parte, y coincidiendo aproximadamente con el periodo de hegemonía teórica del keynesianismo en economía (particularmente de los autores neokeynesianos), asistimos a la irrupción en sociología de la generación de los "compiladores" (Lamo de Espinosa, 2001, p. 31); una generación que, en su vertiente funcionalista, encuentra a su líder indiscutible en la figura de Talcott Parsons y en su apuesta por la doctrina estructural-funcionalista.

Ahora bien, hemos de tener muy presente que la obra de Parsons presenta dos momentos claramente diferenciados. En el primero, el autor se centra en el estudio de la acción social, mientras que en el segundo se orienta hacia el análisis del sistema social. Este corte en su miento ${ }^{2}$ implica "inconsistencias"

2 Corte que, en retrospectiva, quizá venga ya anunciado desde su manuscrito de 1939, inédito en vida, Actor, Situación y Pautas Normativas. Allí afirma: "En cierto sentido, un sistema social tiende a un 'equilibrio estable', a una conservación duradera de sí mismo como sistema y al mantenimiento de un determinado modelo estructural, estático o dinámico. En este sentido es análogo (no idéntico) a un organismo y su tendencia a mantener, a corto plazo, un equilibrio fisiológico o una 'homeostasis', y a seguir, a largo plazo, la curva del ciclo vital" (Parsons, 1939, p. 103, citado en Joas \& Knöbl, 2004, p. 66, cursivas en el original).

3 Habermas (1987) llega a afirmar que "De la gran tentativa de Parsons sólo podremos aprender algo si tomamos en serio su intención y analizamos las instructivas contradicciones en que se ve envuelto en la realización de ese propósito. Parto, pues, de que el problema de cómo poner en relación los conceptos fundamentales de la teoría de la acción y de la teoría de sistemas es, como tal problema de construcción, un problema real" (pp. 285-286). 
(Habermas, 1987, pp. 281-425) o, al menos, una cierta "tensión" (Joas \& Knöbl, 2004, p. 75) que viene parcialmente confirmada por el propio Parsons al afirmar: "Creo que ocurrió un cierto giro cuando desvié un poco mi atención de la preocupación por el problema económicosociológico y comencé a considerar la teoría del sistema social en un sentido 'estructuralfuncionalista"' (Parsons, 1974, p. 216).

El primer Parsons, el accionalista, mantiene una posición teórica muy próxima a la weberia$n a^{5}$ y a la de economistas con un origen teórico más o menos "austriaco" como Schumpeter o Knight. Sin embargo, coincidiendo con su nombramiento en 1946 como Director del Departamento de Relaciones Sociales de Harvard, antiguo Departamento de Sociología, su interés teórico comienza a virar desde la esfera concreta de la acción social hacia la del sistema social (este segundo periodo es, precisamente, el que caracterizamos aquí como plenamente estructural-funcionalista). El mejor ejemplo de este giro sistémico lo encontramos con la publicación, en 1951, de dos de sus obras más conocidas: Hacia una Teoría General de la Acción (Parsons y Shils, 1951), su último texto accionalista; y $E l$ Sistema Social (Parsons, 1951), primer texto plenamente sistémico, y auténtica opera magna de este autor.

Parsons destaca, entre otras cosas, por ser un sociólogo que inicia su carrera intelectual desde una posición muy próxima al pensamiento económico. ${ }^{6}$ Como él mismo señala: "el problema sustantivo más importante de las primeras fases de (...) mi trabajo teórico" remitía a "las relaciones entre la teoría económica y lo que en

4 Joas y Knöbl (2004) concluyen que Parsons "no logró verdaderamente llevar a cabo la síntesis entre teoría de la acción y el funcionalismo" (p. 97).

5 No en vano, el lema con el que inicia su obra $L a$ Estructura de la Acción Social (Parsons, 1937) remite a una afirmación del propio Weber: "Todo conocimiento imaginable de los últimos elementos del quehacer humano está ligado, ante todo, a las categorías de 'meta' y 'medio'" (p. 31).

6 No debemos perder de vista que durante su periodo formativo en Amherst orienta sus estudios hacia la economía. aquel momento consideraba teoría sociológica" (Parsons, 1974, p. 216). Es precisamente tal hecho el que explica, como bien señala Joas (1992), que el asunto clave sobre el que orbita "su gran libro de juventud [En referencia a La Estructura de la Acción Social (Parsons, 1937)] sea la relación entre la economía y la teoría sociológica" (p. 84); y el que le permite acceder a su puesto en Harvard "ante todo para transmitir a los estudiantes sus conocimientos básicos sobre las teorías económicas dominantes en Alemania -estas habían sido en parte objeto de su tesis" (Joas \& Knöbl, 2004, p. 30, cursivas en el original).

Ahora bien, hemos de tener muy presente que sus simpatías en esta esfera concreta de la teoría económica se dirigen, especialmente, tanto hacia el pensamiento económico clásico como hacia la obra del propio Keynes. En tal sentido, por ejemplo, Parsons (1974) pone de manifiesto cómo, al criticar la idea de inestabilidad intrínseca de los "medios simbólicos generalizados de intercambio" (p. 218), se siente "en terreno muy firme" (p. 218). Esto gracias a la "interpretación del dinero como fenómeno institucionalizado; basada, en gran medida, en la autoridad de los economistas clásicos y de Keynes" (p. 218). En cualquier caso, no debemos perder de vista que, con el paso del tiempo, el propio Parsons llega a tomar conciencia de que "el enfoque que estaba adoptando le arrojaba fuera de la prestigiosa disciplina en la que había comenzado su carrera [en referencia a la economía]" (Joas, 1992, p. 86).

\section{Agente individual}

Lo primero que debemos señalar con respecto a la caracterización que llevan a cabo los economistas keynesianos del agente individual es que esta resulta descendiente directa del pensamiento humanista de la escuela neoclásica de Cambridge. Así, sitúan en el centro de su reflexión la capacidad intelectual del agente, abandonan la cuestión del egoísmo en favor del concepto jevonsiano de maximización, y destacan el papel que juegan las expectativas en el proceso de toma de decisiones.

Ahora bien, el análisis de los agentes individuales puede enfocarse de dos maneras 
distintas: la formal, basada en la construcción de modelos pretendidamente no realistas; y la material, que atiende a su efectiva actuación en el mundo. En tal sentido, el modo en que proceden los autores adscritos a estas dos corrientes keynesianas no puede resultar más contrapuesto. Mientras que Keynes y los postkeynesianos parten de la racionalidad material de los agentes e incorporan factores no racionales, los autores neokeynesianos apuestan por establecer un modelo estrictamente formal. Así, la caracterización que lleva a cabo Parsons de los agentes individuales ocupa un espacio intermedio entre las dos posturas por cuanto, si bien destaca el papel de lo no racional (como los postkeynesianos), lo hace desde una perspectiva epistemológica que implica el establecimiento de un modelo formal (como los neokeynesianos).

Keynes y los postkeynesianos consideran que el proceso de formación de expectativas presenta dos componentes principales: el peso de los argumentos y la probabilidad. Resulta evidente, por tanto, que tales autores parten de un modelo de agente que dispone de un conocimiento imperfecto del futuro. Sin embargo, como apunta el propio Keynes al respecto, hemos de diferenciar claramente entre riesgo (que, como en los juegos de azar, es calculable a priori) e incertidumbre (que no lo es porque depende de acontecimientos impredecibles). A Keynes le interesa esta segunda y aclara que:

\footnotetext{
El sentido en que estoy usando el término es el mismo que cuando digo que la previsión de una guerra en Europa es incierta, o que lo es el precio del cobre y la tasa de interés dentro de veinte años, o la obsolescencia de una nueva invención, o la posición de los poseedores de riqueza en el sistema social en 1970. Acerca de esas cosas no hay bases científicas respecto a las cuales formar una probabilidad calculable. Simplemente no sabemos (Keynes, 1937, p. 214).
}

La incertidumbre, por tanto, consiste para Keynes en la incapacidad de un agente para atribuir una determinada probabilidad a un suceso futuro. Pero esta situación desemboca necesariamente en la inacción. ¿Cómo superarla? Mediante mecanismos no racionales como "la imaginación" (Shackle, 1961) o "el hábito ciego y la convención" (Robinson, 1980, p. 219). Si tuviéramos que salir por alguna razón a la calle y hubiéramos de decidir racionalmente si sacar el paraguas cuando hay nubes en el cielo, deberíamos valorar todos los argumentos y probabilidades de cada posible vía de acción; lo que al final podría traducirse en no salir siquiera de casa. Así, como afirma Keynes (1921): "no es siempre racional que una postura prevalezca sobre la otra en nuestras mentes, o incluso que debamos equilibrarlas -más bien será racional dejar a nuestro capricho determinarlo y no desperdiciar el tiempo en el debate-" (p. 32).

En el mismo sentido, en su época accionalista, Parsons afirma que:

\begin{abstract}
El hecho de que se abra al actor un abanico de posibilidades (en relación tanto con los fines como con los medios) en combinación con el concepto de una orientación normativa de la acción, supone la posibilidad de "error", de fracaso en la consecución de los fines o en la elección "correcta" de los medios (Parsons, 1937, p. 84).
\end{abstract}

Tal "posibilidad de error" se ve reducida gracias a la introducción, en su época sistémica, del concepto de expectativas de rol. En este segundo periodo, Parsons (1951) entiende al agente individual como un sistema de personalidad.7 Dicho sistema es influido por otros tres subsistemas: el social, el cultural y el biológico. De este modo, los hábitos o convenciones (por decirlo en términos robinsonianos) que orientan la acción del agente en contextos de incertidumbre provienen del subsistema social y del cultural. El primero pretende contrarrestar la conducta desviada y delictiva mediante mecanismos de control que imponen sanciones para mantener la estabilidad social, mientras que el segundo

7 Aunque ya podemos rastrear referencias a esta aproximación teórica de agente individual como sistema de personalidad en su texto, inédito en vida, de 1939 Actor, Situación y Pautas Normativas, donde afirma: "Este sistema se denominará la personalidad, y lo definiremos como el sistema organizado de la orientación y la motivación de la acción de un actor individual" (Parsons, 1939, p. 7, citado en Joas \& Knöbl, 2004, p. 68). 
influye en la personalidad a través del proceso de socialización. Durante este proceso, los agentes incorporan modelos culturales imperantes, especialmente los valores y las normas. Así, el sistema de personalidad se compone de disposiciones de personalidad que impulsan al actor a buscar la aprobación en sus relaciones sociales, seguir los modelos culturales imperantes y a no adoptar determinados roles negativamente sancionados. De esta doble influencia es de donde emergen las expectativas de rol como mecanismo orientado a reducir la incertidumbre. Tales funcionan, por tanto, como guías para la acción gracias a las cuales el individuo puede actuar en contextos de incertidumbre.

Además, Keynes diferencia entre expectativas a corto y a largo plazo, atendiendo al nivel de confianza y al coste en relación con la incertidumbre. Sin embargo, los autores postkeynesianos no están de acuerdo con tal distinción porque consideran que, ni unas, ni otras, presentan una menor incertidumbre. Con respecto a las expectativas a corto plazo, Robinson (1980) afirma que "en un modelo a corto plazo no hay previsiones correctas. Hay expectativas individuales que no tienen por qué ser coherentes entre sí y que pueden generarse tras haber confundido al agente" (p. 223); a lo que Shackle (1967) añade, además, que, como lo demuestra la historia, "a largo plazo todo es posible" (p. 132).

No obstante, la diferencia entre expectativas a corto y a largo plazo sí les permite diferenciar, tanto a Keynes (1936), como a Kaldor (1939), entre empresarios y especuladores. Los primeros invierten en función de su expectativa de dividendos futuros a largo plazo ya que "la palabra empresa, o espíritu de empresa, es la tarea de prever rendimientos probables de los bienes por todo el tiempo que duren" (Keynes, 1936, p. 144). Por su parte, los segundos buscan beneficios del capital a corto plazo mediante "la actividad que consiste en prever la psicología del mercado" (Keynes, 1936, p. 170). Es en este último sentido en el que Keynes (1936, pp. 142143) introduce su famoso símil entre el proceder especulativo y los concursos de belleza de los periódicos, donde los suscriptores obtienen un premio si logran acertar quién va a alzarse con la victoria.

El resultado de la acción especulativa resulta imposible de predecir ya que no es único, ni está predeterminado por variables económicas fundamentales, sino por rumores o creencias sobre estas (Shackle, 1955). Tal situación es la que caracteriza en economía, por ejemplo, la determinación de la tasa de interés. Según Keynes, esta:
es un fenómeno muy convencional, más que muy psicológico; porque su valor real está determinado en gran parte por la opi- nión que prevalezca acerca del valor que se espera irá a tener. Cualquier nivel de inte- rés que se acepte con suficiente convicción como probablemente duradero, será dura- dero; sujeto, en una sociedad cambiante, por supuesto, a fluctuaciones alrededor del nivel normal esperado debidas a toda clase de motivos (Keynes, 1936, pp. 182-183).

Como resulta evidente, este fenómeno se corresponde, en la esfera estrictamente sociológi$\mathrm{ca}$, con la denominada "profecía autocumplida" que analiza el destacado discípulo de Parsons, Robert K. Merton (1948).

Aunque las expectativas son el elemento fundamental que caracteriza al agente individual en la obra de Keynes y de los postkeynesianos, hay otros dos factores a los que también debemos atender: los animal spirits y la ilusión monetaria. Previamente hemos afirmado que el agente individual puede entregarse a la inacción debido a la incertidumbre asociada al proceso de toma de decisiones, pero también que puede superarla valiéndose de mecanismos no racionales. Uno de los mecanismos remite a la noción de animal spirits. La introducción del concepto por parte de Keynes permite comprender:

\footnotetext{
que gran parte de nuestras actividades positivas dependen más del optimismo espontáneo que de una expectativa matemática, ya sea moral, hedonista o económica. Quizá la mayor parte de nuestras decisiones de hacer algo positivo, cuyas consecuencias completas se irán presentando en muchos días por venir, sólo pueden considerarse
} 
como resultado de la fogosidad ["animal spirits" en el original] -de un resorte espontáneo que impulsa a la acción de preferencia a la quietud, y no como consecuencia de un promedio ponderado de los beneficios cuantitativos multiplicados por las probabilidades cuantitativas- (Keynes, 1936, p. 147).

Previamente hemos aludido a cómo Parsons considera que las disposiciones de personalidad permiten ejecutar la acción individual en un contexto de incertidumbre. Además, hemos explicado cómo su carácter previsible deriva de su relación con los subsistemas social y cultural. Sin embargo, el sistema de personalidad también es influido por el subsistema biológico. Este le otorga, mediante impulsos, la energía necesaria para desarrollar un funcionamiento correcto. Pues bien, son precisamente tales impulsos los que presentan características muy similares a los animal spirits (o "fogosidad") a los que alude Keynes, ya que ambos conceptos remiten, en última instancia, a los componentes no racionales de la conducta. Es más, no debemos perder de vista que los elementos "no racionales e inconscientes" (Parsons, 1951, p. 293) resultan una constante en la obra de Parsons como demuestran las múltiples referencias que introduce en su obra a los trabajos de Freud. ${ }^{8}$ En el mismo orden de cosas podemos recordar también cómo la concepción parsoniana de la personalidad como sistema encuentra, según el propio autor (Parsons, 1951, p. 546), su antecedente más inmediato en los escritos del fundador del psicoanálisis. ${ }^{9}$ Circunstancia que,

8 Por ejemplo, Parsons (1970).

9 Parsons (1951) afirma textualmente: "Puede decirse que la concepción de la personalidad como sistema emergió definitivamente en su trabajo [de Freud]" (p. 546). Así, la influencia de la variante más ortodoxa de la doctrina freudiana pudo haberse traducido en la consideración de Parsons (1970, pp. 78-111) de que el proceso de interiorización de normas y formación del super-yo es el que permite establecer un puente entre la psicología y la sociología; al tiempo que se constituye como el paso decisivo en la formación de la personalidad. No debemos perder de vista que el propio Parsons "se somete a análisis y recurre a otras teorías psicológicas afines de su tiempo para poder explicar los motivos impulsores que, ya en la primera infancia, quedan enraizados en la personalidad y determinan a la persona durante el resto de su vida" (Joas \& Knöbl, 2004, p. 61). en cualquier caso, no le impide criticar a Freud por no haber utilizado el modelo sistémico como "guía definitiva" (Parsons, 1951, p. 546), ni haberlo separado completamente de sus aspectos estrictamente biológicos.

El segundo factor no racional al que alude Keynes es la ilusión monetaria. Tal fenómeno se circunscribe a los trabajadores y consiste en su incapacidad para manejar complejas variables económicas. Según el autor, la racionalidad de los trabajadores se ve comprometida al no poder diferenciar correctamente entre salarios nominales y reales; lo que puede desembocar en que acepten aumentos del salario en unidades monetarias que, en realidad, suponen una reducción de su poder adquisitivo. Robinson (1937, p. 3-4) critica este supuesto y destaca el papel que pueden jugar, en tal sentido, los sindicatos para resolver dicho problema.

En cualquier caso, no debemos considerar que el fenómeno de la racionalidad limitada caracteriza únicamente a los trabajadores (aunque, concretamente, sí el fenómeno de la ilusión monetaria). Como acabamos de señalar, las expectativas empresariales también resultan poco racionales debido al contexto de incertidumbre en el que deben elaborarse y a la incorporación de la doctrina de los animal spirits. No obstante, trabajadores y empresarios deben ser considerados como agentes maximizadores, ya que mientras los primeros pretenden maximizar sus sueldos, los segundos actúan impulsados "por el deseo de obtener un máximo de ganancias presentes y futuras" (Keynes, 1936, p. 77).

Parsons (1937) coincide con Keynes y los postkeynesianos en imputar tal carácter maximizador al agente ya que, al menos en su época accionalista, considera que su comportamiento puede ser analizado desde la estructura del acto-unidad. Marco teórico que se caracteriza por una "orientación normativa" (Parsons, 1937, p. 83) donde un agente maximizador persigue determinados fines a través del uso de medios alternativos en la medida en que la situación se lo permite. Hasta aquí, tal aproximación no aporta grandes novedades con respecto a la clásica definición de acción económica establecida 
por Robbins (1932, p. 15). Sin embargo, Parsons (1937, p. 86) va más allá y agrega dos nuevos elementos: las condiciones situacionales y las normas orientadoras. Esta doble incorporación le permite posteriormente, en su época sistémica, considerar al agente como un sistema de personalidad influido por el sistema social y cultural (de donde, como acabamos de señalar, extrae sus orientaciones y motivaciones para la acción).

La existencia de dos marcos teóricos referidos al agente individual (la estructura del acto-unidad y el sistema de personalidad) no hace sino poner de manifiesto el corte teórico, al que hemos hecho referencia al inicio del artículo, entre el Parsons accionalista y el sistémico; $y$, concretamente en el segundo periodo, aproximar su modelo de agente a lo que Garfinkel (1968, p. 82) denomina "idiota cultural". ${ }^{10}$

Como podemos comprobar, la caracterización del agente individual que llevan a cabo, tanto Keynes y los postkeynesianos en economía, como Parsons en sociología, presenta un carácter maximizador acompañado de una limitada capacidad de cálculo, y está fuertemente influida por componentes no racionales. Sin embargo, Parsons (1951), aunque, como acabamos de señalar, comparte con Keynes y los postkeynesianos los principales elementos caracterizadores del agente individual, también afirma que las auténticas unidades a las que debemos atender a efectos analíticos son las "relaciones interactivas y los roles recíprocos de las partes" (p. 394), y no los individuos propiamente dichos. Tal giro parsoniano hacia la incorporación de modelos formales en sus textos del periodo sistémico (que se inicia con la introducción de la noción de sistema de personalidad) puede explicarse como una consecuencia no prevista de la apuesta que el autor realiza, ya desde su época accionalista, por el realismo

10 El propio Garfinkel (1968) se encarga de aclarar que por "idiota cultural" comprende "al hombre-en-la-sociedaddel-sociólogo, hombre que produce las características estables de la sociedad al actuar de acuerdo con las alternativas preestablecidas y legítimas de la acción suministradas por la cultura común" (p. 82). analítico ${ }^{11}$ frente al realismo crítico por el que se decantan, según Lawson (1994, p. 507), tanto Keynes, como los economistas poskeynesianos.

Por su parte, uno de los principales supuestos sobre los que se erige el modelo formal de agente individual -elaborado por los economistas neokeynesianos- consiste en atribuirle un comportamiento maximizador. Pero no sólo eso. El propio Samuelson (1969, p. 12) afirma que, para que los sistemas de los que se valen los economistas puedan ser considerados científicos, deben incorporar el axioma de ergodicidad. Este implica que el conocimiento de los agentes "sobre el futuro supone la proyección de promedios calculados que se basan en el pasado y/o en muestras representativas actuales y/o en datos de series temporales para eventos venideros" (Davidson, 1982, p. 90). Como podemos comprobar, el agente neokeynesiano, además de maximizador, debe ser comprendido como una figura más próxima a un actuario de seguros que no cometiera errores (y que además dispusiera de toda la información "relevante" a la hora de tomar decisiones) que a una persona real. Aunque Samuelson no considera que los agentes se comporten realmente así, incorpora tal postulado debido a la utilidad predictiva que le imputa. Esta es una de las razones por las que caracterizamos su aproximación epistemológica a la economía como formalismo analítico.

El comportamiento maximizador se atribuye a consumidores y a empresarios. Para analizar la conducta de los primeros, Samuelson (1938) se remite a la doctrina de la "preferencia revelada". Según tal, resulta posible descubrir las prioridades de los consumidores atendiendo a sus elecciones efectivas a la hora de adquirir productos en un entorno caracterizado por la restricción presupuestaria. El autor parte del carácter transitivo de las preferencias y de la consistencia del

11 La primera referencia explícita a este "realismo analítico" la encontramos en: Parsons (1937, p. 887). Dicho realismo analítico parsoniano significa, como acertadamente señala Joas (1992), que, "aunque se mantiene el propósito de explicar la realidad, [éste] sólo puede alcanzarse aislando los fenómenos analíticos específicos" (p. 72). 
comportamiento del consumidor, concluyendo que "es posible derivar hipótesis significativas sobre las funciones de demanda de los consumidores desde la premisa de que su comportamiento maximiza una escala de preferencias ordinales de cantidades de consumo, bienes y servicios" (Samuelson, 1983, p. 22). Del mismo modo, también señala cómo el comportamiento de los empresarios "está dirigido hacia la maximización de beneficios con ciertas implicaciones para la minimización de los costes" (Samuelson, 1983, p. 21).

En cualquier caso, Samuelson (1952), aunque reconoce que "no hay controles absolutos contra el error humano" (p. 64), argumenta que, si se siguen dichas premisas, ambos comportamientos resultarán susceptibles de ser anticipados. Y es precisamente con tal propósito que los autores neokeynesianos elaboran la teoría de las expectativas adaptativas. Según esta, los agentes económicos, al hacer sus predicciones, tienen en cuenta exclusivamente datos procedentes del pasado, por lo que sus errores resultan previsibles. Para llegar a tal conclusión, sin embargo, deben eliminar de sus análisis factores no racionales como los animal spirits, debido a la dificultad que entraña su operativización en un entorno metodológico tendente al uso de herramientas matemáticas.

\section{Organizaciones}

$\mathrm{Si}$ optamos, como hacen los autores mencionados, por comprender las organizaciones como agentes corporados, debemos partir del hecho de que estas se enfrentan con dos tipos de restricciones. En la esfera intraorganizativa han de lidiar con problemas derivados de la necesaria coordinación de las acciones individuales; mientras que en la interorganizativa deben ser capaces de operativizar su acción colectiva. Así, para analizar la doctrina defendida por Parsons y por los economistas keynesianos, hemos de atender, por una parte, al surgimiento y función de las organizaciones; $y$, por otra, a las relaciones que estas mantienen entre sí.

El análisis que los economistas keynesianos llevan a cabo, respecto a las organizaciones en su vertiente estrictamente intraorganizativa, pasa por ser uno de los elementos a los que menor atención prestan. Sus trabajos inciden, tanto en su decidida apuesta por la división del trabajo, como en dar por consumado el proceso de separación entre las esferas de propiedad y gestión dentro de una empresa. Con respecto al primer elemento, Keynes (1936) se limita a afirmar las ventajas "reales y sustanciales" (p. 300) de la división internacional del trabajo; mientras que Samuelson (1970, p. 56), apoyándose en el clásico ejemplo de Adam Smith de la fábrica de alfileres, destaca el aumento de productividad que esta genera.

Por otro lado, Parsons, que sí se detiene a analizar con cierta minuciosidad las dinámicas intraorganizativas valiéndose de su modelo AGIL, ${ }^{12}$ considera que la división del trabajo constituye el concepto clave de la acción instrumental, y se remite, en tal sentido, a las investigaciones desarrolladas por Adam Smith y "sus seguidores en la tradición utilitaria, especialmente económica" (Parsons, 1951, p. 70). Además, Parsons (1956a) también afirma que "la existencia de organizaciones (...) es una consecuencia de la división del trabajo en la sociedad" (pp. 65-66); es decir, el desarrollo de las organizaciones "es el mecanismo principal mediante el cual, en una sociedad altamente diferenciada, es posible 'hacer las cosas', lograr objetivos que se sitúan más allá del alcance individual y hacerlo bajo condiciones que proporcionan una cierta maximización de la eficacia" (Parsons, 1956b, p. 225). Así, la

12 Podemos encontrar tal modelo, por ejemplo, en: Parsons y Smelser (1956, p. 242). El esquema teórico AGIL se basa, según confiesa el propio Parsons (Parsons, Bales \& Shils, 1953, p. 64), en los trabajos previos desarrollados por Robert Bales. Para el análisis específico de las organizaciones por parte de Parsons, véase: Parsons (1956a; 1956b). La presencia del modelo AGIL en estos dos textos concretos sobre las organizaciones se ve refrendada al afirmar Parsons (1956b): "El esquema que hemos presentado se caracteriza por una cierta simetría formal. El sistema de valores de la organización trata de definir y legitimar su objetivo. Cada una de las otras tres esferas, los mecanismos de adaptación, los mecanismos de consecución de objetivos operativos y la integración de la organización, están regulados por subvalores que gobiernan cada uno de estos tres aspectos de la función organizacional" (p. 228). 
movilización de poder para lograr los objetivos se constituye como "el fenómeno central" (Parsons, 1956b, p. 225), el "foco central operativo" (Parsons, 1956b, p. 227), de toda organización. Es en tal sentido en el que debemos comprender la afirmación de Parsons de que el poder remite, antes que nada, a "la capacidad generalizada para movilizar recursos en aras a alcanzar los fines de un sistema" (Parsons, 1956b, p. 225).

Con respecto a la separación entre las esferas de propiedad y gestión, Keynes (1936, p. 137) llama la atención sobre el hecho de que la propiedad de las primeras empresas, a diferencia de las de su tiempo, remitía a los propios fundadores, sus amigos o asociados; mientras que Samuelson y Nordhaus (2005) argumentan que "el primer paso para comprender el comportamiento de las grandes empresas [de su propia época] es darse cuenta de que son en su mayor parte de 'propiedad púbica"' (p. 189). Con tal afirmación pretenden destacar cómo las participaciones en la propiedad (acciones) pueden repartirse entre múltiples inversores, de tal modo que "normalmente la propiedad y el control están separados" (Samuelson \& Nordhaus, 2005, p. 189). Ambos autores advierten, además, que tal situación puede suponer la emergencia de dos tipos de conflictos de intereses: por una parte, los directivos pueden aprovechar su posición para elevar sus propios salarios en detrimento de los accionistas; $y$, por otra, pueden retener beneficios a fin de ampliar la empresa sin una justificación económica suficiente.

Por su lado, Parsons (1951) define las empresas como "subcolectividades instrumentalmente orientadas" (p. 146), y distingue tres tipos de roles asociados: roles técnicos diferenciados, roles políticos y roles administrativos o de instrumentación. Los directivos de las empresas, en tanto que tales, desempeñan roles políticos por cuanto adoptan "decisiones con respecto a las orientaciones hacia una meta de la organización" (Parsons, 1951, p. 146). Pero, ¿cuál es esa meta? Al menos en el Sistema Social, el beneficio: en una empresa, según señala este mismo autor, "el beneficio tiene la máxima primacía"; se constituye como la "obligación suprema de los altos roles directivos" (Parsons, 1951, p. 246).
Afirmamos que esta es la doctrina que defiende Parsons "al menos" en el Sistema Social, porque años más tarde afirma que:

para la empresa de negocios, el retorno monetario es una medida primaria y un símbolo de éxito; y, por lo tanto, parte de la estructura de objetivos de la organización. No obstante, no puede ser el objetivo principal de la organización, ya que la obtención de beneficios no es en sí misma una función de la sociedad como sistema (Parsons, 1956a, p. 68).

A lo que posteriormente agrega que:

la empresa comercial se rige por los valores de la racionalidad económica: la maximización de la producción con un coste mínimo (en sentido económico). El fin institucionalizado de la empresa es la producción. Sin embargo, en una economía de mercado, el rendimiento financiero se constituye como una condición para el funcionamiento continuo y como un símbolo central del éxito. La referencia a la función social [es decir, a la producción] es primaria; la referencia al beneficio mercantil es secundaria, pero muy importante (Parsons, 1956b, p. 230, cursiva en el original).

En cualquier caso, a Parsons (1951, p. 509) tampoco se le escapa el contraste que existe entre lo que él denomina la era de los "negocios independientes" y la época de la empresa burocratizada. Tomando como ejemplo el caso concreto de los Estados Unidos, diferencia dos periodos cuyo corte establece, aproximadamente, al final de la Primera Guerra Mundial. En el primero, el empresario individual era el "propietario-director" y "las organizaciones eran lo suficientemente simples como para ser creadas y dirigidas casi ad hoc por el empresario mismo" (Parsons, 1951, p. 509). Desde entonces, sin embargo, el ejecutivo "ha ocupado el lugar del primitivo empresario" (Parsons, 1951, p. 509). Parsons (1956b) califica de "notable" el hecho de que en su propia época se haya producido una "marcada disminución en la legitimación del control basada en la propiedad de los activos de capital" (p. 235) acompañada de "un aumento correspondiente del control basado en la 
efectividad de una administración, la mayoría de cuyo personal son formalmente "empleados"' (p. 235). Desde tal punto de vista, los posibles conflictos a los que alude Samuelson pueden ser analizados como disfunciones dentro del subsistema orientado a definir y alcanzar metas del modelo AGIL aplicado a una empresa. ${ }^{13}$

En la esfera interorganizativa, todos los economistas keynesianos coinciden en definir al Estado como un agente económico legítimo. Hasta aquel momento, la mayor parte de los autores anglosajones consideraba que el Estado debía limitarse a establecer las reglas del juego económico. Sin embargo, los keynesianos, sin abandonar dicha doctrina, también contemplan la posibilidad de que actúe directamente en el mercado a fin de implementar políticas anticíclicas a través de "una influencia orientadora sobre la propensión a consumir, a través de su sistema de impuestos, fijando la tasa de interés y, quizá, por otros medios" (Keynes, 1936, p. 332).

En el mismo sentido, Samuelson y Nordhaus (2005, pp. 195-196) aluden a seis mecanismos de intervención estatal orientados a corregir los abusos en situaciones de "poder de mercado": la política antimonopolio, los estímulos a la competencia, la regulación, la propiedad del Estado, los controles de precios y los impuestos. Los tres primeros y el último, los más frecuentes, caen dentro de la tradicional función institucional imputada -hasta aquel momento- al Estado. Sin embargo, el cuarto y el quinto resultan menos frecuentes en economías como la de los Estados Unidos de la época y destacan por poner de relieve la capacidad de agencia del Estado en la esfera económica. Como defiende Coddington (1976), el keynesianismo "hidráulico" (neokeynesianismo en nuestra terminología) es una teoría económica:

en la que sólo hay una agencia que lleva a cabo actos deliberados de elección, esa

13 Por otro lado, por ejemplo, Parsons (1956a) también comprende el fenómeno concreto de la restricción de la producción como un conflicto analizable desde el punto de vista del modelo AGIL; en concreto, como "un caso de fracaso relativo de integración" (p. 80, nota al pie 9). agencia es el "gobierno". Y es la creencia de que efectivamente existe una relación estable entre los distintos flujos globales de la economía la que proporciona su base al "gobierno" para perseguir sus objetivos políticos en cuanto al nivel general de la actividad económica; y, por lo tanto, (...) al nivel de empleo (Coddington, 1976, p. 1265).

Esta posición neokeynesiana, que puede derivar de la inclusión del concepto de equilibrio walrasiano (basado en la existencia de un sistema ergódico cuyos elementos establecen relaciones de mutua dependencia entre sí) en la esfera epistemológica, presenta claros paralelismos con la doctrina defendida por Talcott Parsons. En tal sentido, por un lado, el sociólogo estadounidense afirma que los cuatro subsistemas del sistema social se relacionan entre sí buscando establecer una situación de equilibrio; pero, por otro, añade también que:

\footnotetext{
Una economía es un tipo especial de sistema social. Es uno de los subsistemas funcionales más inclusivos de la sociedad, que se diferencia de otros subsistemas por la especialización en la función adaptativa de la sociedad. Es uno de cuatro subsistemas diferenciados sobre una base de afinidad, y debe distinguirse de cada uno de los demás. También debe distinguirse de todas las colectividades concretas, que, cualquiera que sea su primacía funcional, son siempre multifuncionales. Como sistema social, la economía tiene todas las propiedades de tal sistema (Parsons y Smelser, 1956, pp. 306307, cursivas en el original).
}

Ahora bien, si partimos de las dos premisas (la necesaria relación que debe establecerse entre los cuatro subsistemas del sistema social para alcanzar una situación de equilibrio y la concepción de la economía como un tipo especial de subsistema), la acción del Estado, que procede del subsistema político, en el subsistema económico (y del último sobre el subsistema político) puede ser considerada como legítima siempre que se oriente a establecer o reestablecer una situación de equilibrio general. Así, Parsons, en su obra conjunta con Smelser, presenta un esquema en el que explicita el doble proceso de influencia que puede producirse entre 
la economía (en tanto que subsistema social orientado a la adaptación) y la política (entendida como subsistema social cuya función remite a la definición y consecución de fines).

Por otro lado, no debemos perder de vista que el propio Parsons (1956b) establece cuatro formas principales de organización "atendiendo al tipo de fin o función sobre el cual están organizadas" (p. 228) prioritariamente. Según el autor, en primer lugar, nos encontramos con las organizaciones orientadas a la producción económica (como la empresa de negocios). Ahora bien, el concepto de "producción económica" debe ser comprendido "en un sentido económico completo como "agregadora de valor" (Parsons, 1956b, p. 228). No se limita, pues, "a la producción física, por ejemplo, a la fabricación" (Parsons, 1956b, p. 228). En segundo lugar, las organizaciones orientadas a objetivos políticos se centran en "la generación y asignación de poder en la sociedad: esta categoría incluye a la mayoría de los órganos de gobierno, pero en una sociedad como la nuestra, participan también otras organizaciones" (Parsons, 1956b, p. 229). Entre ellas, Parsons (1956b) incluye explícitamente a los bancos, en tanto que "la asignación de poder de compra a través de la creación de crédito" (p. 229) constituye un "ejercicio de poder" (p. 229). En tercer lugar, las organizaciones integradoras "contribuyen principalmente a la eficiencia, que no a la eficacia, a nivel social" (Parsons, 1956b, p. 229). Se centran, tanto en la "gestión de conflictos" (Parsons, 1956b, p. 229), como en "la orientación de la motivación para el cumplimiento de las expectativas institucionalizadas" (Parsons, 1956b, p. 229). Nos encontramos aquí, por ejemplo, con los tribunales de justicia o los partidos políticos. Finalmente, las organizaciones orientadas al mantenimiento de patrones desarrollan funciones "principalmente 'culturales', 'educativas' y 'expresivas"' (Parsons, 1956b, p. 229). Caen dentro de la última categoría organizaciones como las iglesias o las escuelas.

Como podemos comprobar, todos estos tipos de organización encuentran su razón de ser en la capacidad que demuestran para cumplir con las funciones atribuidas a un sistema social en su conjunto. Parsons (1956b) llega, incluso, a afirmar que el logro del fin específico imputado a cada uno de tales tipos de organizaciones "es, al mismo tiempo, el desempeño de un tipo de función en nombre de un sistema más inclusivo, la sociedad" (p. 238). Por lo tanto, la acción del Estado, en tanto que organización orientada a objetivos políticos, sobre las organizaciones orientadas a la producción económica puede ser considerada legítima siempre que, con la misma, se pretenda facilitar el desarrollo de las funciones normales atribuidas a un sistema social en su totalidad; es decir, a la sociedad. $\mathrm{Ni}$ que decir tiene que, además, la inclusión del sistema bancario en la categoría de organizaciones orientadas a objetivos políticos no hace sino poner de manifiesto la fuerte imbricación -cuya existencia Parsons defiende- que existe entre el subsistema económico y el subsistema político. Parsons señala, en el mismo sentido, que "un determinante principal del tipo de organización es el tipo de intercambio que opera entre el subsistema social en el que la organización está principalmente anclada y el subsistema contiguo" (Parsons, 1956b, p. 230).

Por otra parte, según Keynes (1936, p. 267), "la psicología de los trabajadores" y "la política de los patronos y los sindicatos obreros" (Keynes, 1936, p. 267) establecen discontinuidades en las variaciones salariales con relación a los precios que pueden favorecer un aumento de la demanda agregada; que, a su vez, puede repercutir en una mayor creación de empleo.

En el mismo orden de ideas, como hemos señalado previamente, Robinson argumenta que la labor de los sindicatos puede resultar beneficiosa para los trabajadores, en tanto que favorece una adecuada comprensión -por parte de éstos últimos- de sus intereses reales. Sin embargo, la misma autora (Robinson, 1937, p. 39) también se muestra escéptica en cuanto a la capacidad de los mismos sindicatos para actuar de un modo coordinado, ya que cada una de tales organizaciones está fundamentalmente interesada en obtener el máximo de beneficios para sus propios afiliados.

Desde la perspectiva parsoniana, y sin negar nunca el posible "conflicto latente de intereses 
entre dirección y trabajo" (Parsons y Smelser, 1956, p. 149), la labor de los sindicatos se amplía hasta incluir también el desarrollo de funciones positivas de cara a mantener el orden social, dado que dichas organizaciones, al tiempo que "protegen los 'intereses' del trabajador" (Parsons y Smelser, 1956, p. 149), desarrollan funciones semi-rituales que logran "integrar al trabajador individual y su hogar en una colectividad más amplia; pertenencia que mejora [,además,] su sentimiento de autoestima y confianza" (Parsons y Smelser, 1956, p. 148). Ahora bien, afirmar la protección de los "intereses" del trabajador por parte de los sindicatos en ningún caso supone impugnar la relevancia que presenta el concepto de remuneración basada en la productividad marginal, pese a que "la posición del sindicato y la interposición de la negociación colectiva entre el trabajador individual y la organización empleadora representan un foco importante de limitación de este principio" (Parsons, 1956b, p. 232).

La actitud favorable que manifiestan Keynes y los postkeynesianos respecto a los sindicatos no es, sin embargo, compartida por los economistas neokeynesianos. Los últimos, aunque constatan la existencia de rigideces entre salarios y precios, consideran que estas pueden constituirse, precisamente, como causantes del desempleo. Tal posición también puede ser explicada atendiendo a la inclusión del concepto de equilibrio walrasiano en su epistemología, ya que, desde esta perspectiva, dichas rigideces dificultarían el establecimiento de relaciones fluidas de mutua dependencia entre los elementos del sistema económico.

Finalmente, todos los economistas keynesianos, al estudiar las relaciones entre empresas, coinciden en afirmar que "la mayoría de las industrias son imperfectamente competitivas" (Samuelson y Nordhaus, 2005, p. 190). Sin embargo, la explicación de tal fenómeno difiere entre los autores de ambas corrientes. Así, los postkeynesianos, con Robinson (1933) a la cabeza, afirman que dicha situación se produce porque cada empresa presenta una curva individual de demanda; es decir, actúa como un monopolio que oferta su propio producto diferenciándolo de todos los demás (gracias, tanto a la falta de información o a las preferencias de los consumidores, como a la ubicación o la publicidad). En general, dichas empresas, además, tienden a reducir los salarios reales, a menos que los sindicatos lo impidan; aunque, como reconoce la propia Robinson (1953-1954, p. 98), existen casos donde esto no sucede e, incluso, pueden observarse aumentos.

Frente a dicha explicación, los autores neokeynesianos, como Samuelson y Nordhaus (2005, p. 183), remiten la preeminencia de la forma de competencia imperfecta a tres factores, que recuerdan mucho a la doctrina marshalliana precedente: la existencia de economías de escala, las barreras de entrada y la interacción estratégica (situación que se produce cuando los planes de cada empresa dependen de la conducta de sus competidoras).

En la misma línea, Parsons (1951) remite el análisis de las agrupaciones de empresas de un mismo sector al término "complejo ecológico instrumental" (p. 146); y pone de relieve como dichas agrupaciones pueden llegar a constituirse como "grupos de complejos" susceptibles de actuar y de ser analizados individualmente. Es más, alude explícitamente a los "inevitables elementos monopolísticos presentes en la organización industrial a gran escala" (Parsons y Smelser, 1956, p. 149). Así, desde la perspectiva analítica del modelo AGIL de Parsons, las tres razones que esgrimen Samuelson y Nordhaus para explicar las formas imperfectas de competencia pueden ser comprendidas como mecanismos propios del subsistema adaptativo que hacen suyas las empresas individuales, en tanto que complejos ecológicos instrumentales, a fin de sobrevivir en el mercado.

Pero Samuelson y Nordhaus (2005, p. 190) van aún más allá y subrayan también el hecho de que las grandes corporaciones son responsables de la mayor parte de la investigación y el desarrollo que se lleva a cabo en esta época. Haciendo suyas las intuiciones originales de Schumpeter, ponen de manifiesto cómo, en no pocas ocasiones, las innovaciones más importantes proceden de monopolios consolidados; 
aunque, añaden, la situación podría modificarse en el futuro.

La doctrina de Parsons resulta particularmente afín a la defendida por los economistas neokeynesianos en este punto concreto. Así, el autor destaca cómo "el técnico muy formado y especializado ha adquirido un lugar estratégico en la estructura de la industria que es muy diferente del inventor 'ad hoc' de los primeros tiempos, cuyas invenciones eran más o menos completas" (Parsons, 1951, p. 509); de tal modo que "la institucionalización del cambio científico y tecnológico ha llevado a una serie compleja de repercusiones dentro del mismo complejo instrumental que ha alterado fundamentalmente su estructura" (Parsons, 1951, p. 509).

\section{Instituciones}

La última categoría a la que vamos a referirnos para llevar a cabo esta comparación remite a las instituciones; a las que definimos como "reglas constitutivas" (Searle, 1995, p. 45). Es decir, como pautas que "no sólo regulan, sino que crean la posibilidad misma de ciertas actividades" (Searle, 1995, p. 45). Así, atendemos a cuatro de sus características principales. En primer lugar, verificamos si se defiende una concepción natural de las instituciones que remita su génesis y lógica de funcionamiento a la propia condición humana. En segundo lugar, examinamos su carácter general, tanto a nivel diacrónico, fijándonos en su evolución histórica; como sincrónico, comprobando si se incluyen instituciones formales y no formales. En tercer lugar, aludimos a su surgimiento espontáneo como producto no deliberado de la acción humana. Y, finalmente, tratamos de las posibles relaciones conflictivas que pueden establecerse entre ellas. Es decir, de la existencia de potenciales contradicciones entre las funciones que se les imputan.

Sin embargo, antes de continuar hemos de hacer una advertencia. Las reflexiones que presentamos a continuación sobre las instituciones en el pensamiento económico keynesiano se centran casi exclusivamente en la obra del propio fundador de la escuela. Esto es así por dos razones. En primer lugar, porque apenas existen aportaciones procedentes de la corriente neokeynesiana. El motivo puede remitir al hecho de que la mayoría de tales autores operan epistemológicamente estableciendo un modelo de sistema económico cerrado que hace abstracción de ellas. En segundo lugar, porque las reflexiones de los autores postkeynesianos, si bien incorporan como factor relevante a las instituciones, salvo en el caso específico de Kaldor, no aportan novedades teóricas sustantivas respecto a la doctrina original expuesta por Keynes.

El último (Keynes, 1936, p. 102) apunta ocho posibles motivos que pueden llevar a los individuos a no consumir todos sus ingresos: mantener una reserva frente a contingencias imprevistas; hacer frente a la vejez, ahorrar para la educación familiar o el mantenimiento de dependientes; disfrutar de interés y reconocimiento debido a un mayor consumo real en el futuro; disfrutar de un aumento gradual de los gastos; beneficiarse de un cierto sentido de independencia; llevar a cabo proyectos especulativos o de negocios; legar una fortuna; y, finalmente, la pura avaricia. Pero Keynes (1936, p. 103) también atiende a las razones que pueden mover a los agentes a establecer un nivel de consumo superior al de sus ingresos: el disfrute, la imprevisión, la generosidad, el error, la ostentación y, finalmente, la extravagancia.

Por otro lado, según Keynes (1936), el Estado también tiende tanto a retener ingresos como a gastar por encima de los mismos por "motivos muy semejantes, pero no idénticos, a los que impulsan a los individuos" (p. 103). Los motivos del Estado para el ahorro son: el motivo empresa: "asegurar recursos para efectuar mayores inversiones de capital sin tener que incurrir en deuda, ni obtener más capital del mercado" (Keynes, 1936, p. 103); el motivo liquidez: "asegurar recursos líquidos para enfrentarse a las emergencias, las dificultades y las depresiones" (Keynes, 1936, p. 103); el motivo mejoramiento: "asegurar un ingreso en aumento gradual que, incidentalmente, pueda proteger a la gerencia 
contra la crítica" (Keynes, 1936, p. 103); y "el motivo prudencia financiera y el afán de sentirse seguro haciendo una reserva financiera que exceda del costo de uso y del suplementario" (Keynes, 1936, p. 103). Por su parte, las razones que pueden favorecer el déficit estatal pueden remitir, por ejemplo, al pago del seguro de desempleo o de las pensiones.

Sin embargo, lo que aquí nos interesa no es tanto atender a estos posibles motivos (individuales y estatales), sino mostrar cómo el elemento más relevante del análisis institucional que desarrolla Keynes sobre dichas razones remite a su afirmación de que:

$$
\begin{aligned}
& \text { la fuerza de todos estos motivos variará } \\
& \text { enormemente, según las instituciones y } \\
& \text { la organización de la sociedad económica } \\
& \text { que supongamos, según los hábitos for- } \\
& \text { mados por la raza, la educación, los con- } \\
& \text { vencionalismos, la religión y las corrientes } \\
& \text { morales; según las esperanzas y la expe- } \\
& \text { riencia, según la escala y técnica del equi- } \\
& \text { po productor y según la distribución de la } \\
& \text { riqueza y los niveles de vida establecidos } \\
& \text { (Keynes, 1936, pp. 103-104). }
\end{aligned}
$$

Como podemos comprobar, Keynes defiende que las instituciones son, a un tiempo, generales y naturales. Son generales, por una parte, porque se incluyen instituciones formales (como "la organización de la sociedad económica" por parte del Estado), e informales (como los "hábitos" o los "convencionalismos"); y por otra, porque se parte de su variabilidad histórica. Como señala el propio Keynes, la Teoría General no trata, sino ocasionalmente, sobre los cambios sociales. Sin embargo, en una de sus escasas referencias en tal sentido, alude a la no existencia de un estándar fijo de liquidez, ya que aquel se modifica "de tiempo en tiempo" (Keynes, 1936, p. 151) y depende "de las prácticas sociales y de las instituciones" (Keynes, 1936, p. 151). Como resulta patente, tal afirmación supone, siquiera implícitamente, la existencia de modificaciones institucionales de carácter diacrónico.

Parsons coincide con la consideración de las instituciones como un fenómeno general ya que, al igual que Keynes, también afirma que pueden ser tanto formales como informales; y que vienen sujetas a una evolución histórica. El autor (Parsons, 1951, p. 58) establece tres tipos de instituciones: relacionales, que definen expectativas de rol recíprocas con independencia del contenido del interés; regulativas, que definen los límites de la legitimidad en la persecución de intereses privados con respecto a metas y medios; y culturales, que definen las obligaciones de aceptar las pautas culturales, convirtiendo la aceptación privada en deber institucionalizado. El segundo tipo, las instituciones regulativas, comprende, tanto instituciones formales como informales (Parsons, 1951, pp. 134-135). El criterio de diferenciación entre ambas formas remite a cómo, mientras que en las instituciones informales las sanciones quedan en manos privadas (y este mecanismo se constituye como el fundamento básico de control social en todas las sociedades próximas a la institucionalización de los roles en general), en las formales (siempre susceptibles de interpretación), los agentes deben atender a las obligaciones específicas de cada uno de los roles, así como a sus mecanismos de refuerzo.

Por su parte, la evolución histórica de las instituciones en la obra de Parsons (1971) alude a un modelo unilineal que comprende tres tipos de sociedad a modo de estadios: primitiva, poco diferenciada y donde prevalecen criterios adscriptivos de organización social; arcaica, donde se diferencia la función secular de la religiosa gracias a la aparición de la escritura y donde pueden identificarse tres niveles de estratificación social (gobernantes, administradores y trabajadores); y moderna, donde las cuatro funciones del sistema social (modelo AGIL) se presentan claramente diferenciadas. Parsons sitúa en la cúspide de este proceso histórico a la sociedad occidental; y, muy particularmente, a la estadounidense de su tiempo.

Por su parte, Keynes defiende el carácter natural de las instituciones en tanto que su génesis y lógica de funcionamiento obedecen a la naturaleza humana (así lo demuestran sus reiteradas referencias a modificaciones conjuntas 
de carácter psicológico e institucional). En tal sentido, podemos destacar la vinculación directa que establece entre la naturaleza humana y el funcionamiento de las instituciones al afirmar que:

\begin{abstract}
Una valoración convencional que se establece como resultado de la psicología de masa de gran número de individuos ignorantes está sujeta a modificaciones violentas debidas a un cambio violento en la opinión como consecuencia de factores que en realidad no significan gran cosa para el rendimiento probable (Keynes, 1936, p. 141).
\end{abstract}

Como aclara a continuación el propio autor, podría pensarse que la función que desarrollan los agentes más expertos que el inversor promedio en el mercado consiste en corregir tales caprichos. No es así. En realidad, pretenden precisamente todo lo contrario a fin de obtener beneficios. En cualquier caso, el funcionamiento del mercado, en tanto que institución, responde a las acciones de los agentes; ya sean estos legos o especialistas.

Con respecto al carácter natural de las instituciones, debemos recordar cómo Parsons (1951) remite la diferencia que establece entre sus tres formas institucionales al hecho de que el sistema social "es esencialmente una trama de relaciones interactivas" (p. 51). Por lo tanto, las instituciones centrales pueden ser comprendidas como aquellas "directamente constitutivas de las pautas de esas relaciones mismas" (Parsons, 1951, p. 51). Así, es la estructura de la relación social como tal la que define la centralidad de cada tipo de institución; relegando a un segundo plano a las de tipo regulativo y a un espacio periférico a las culturales. En cualquier caso, todas ellas, ya sea de un modo mediato o inmediato, remiten, en su origen, a la naturaleza humana; y pueden ser consideradas, por tanto, como naturales en el sentido que aquí otorgamos al término.

Acabamos de afirmar que Keynes defiende que la lógica de las instituciones responde a la naturaleza humana. Sin embargo, esto no supone necesariamente que estas emerjan como un producto deliberado de la acción de los agentes, ni que las funciones que se les imputan no resulten contradictorias. Por lo tanto, debemos centrarnos en las otras dos características a las que venimos refiriéndonos: su carácter espontaneo y conflictivo.

Con respecto al primero, no hemos encontrado referencias explícitas en la obra de Keynes que nos permitan afirmar que defiende dicha doctrina. Por su parte, desde la perspectiva parsoniana, este carácter espontaneo de las instituciones queda desplazado a un segundo plano. Según el autor, el teorema de la integración institucional se basa en la "integración de elementos motivacionales y culturales o simbólicos conjuntados en una cierta clase de sistema ordenado" (Parsons, 1951, p. 36). Así, Parsons (1951) afirma que: "en cualquier sistema dado de relaciones interactivas las pautas tienen que haber sido seleccionadas entre estas posibilidades de manera que sean compatibles con la estabilidad del proceso de interacción" (p. 52). De tal modo, la espontaneidad en el surgimiento de instituciones queda circunscrita al espacio de posibilidades previo que delimita el propio sistema social.

Con respecto al carácter conflictivo de las instituciones, Keynes resulta mucho más explícito. Según confiesa él mismo, el motivo que le mueve a escribir la Teoría General no es otro que establecer una nueva doctrina económica que dé cuenta de los acontecimientos económicos de su propio tiempo; acontecimientos que remiten a una situación de equilibrio estable, pero sin pleno empleo. Como resulta patente, esta situación no puede caracterizarse sino como una situación conflictiva entre las funciones atribuidas a dos instituciones: el Estado y el mercado.

Sin embargo, Parsons no comparte esta doctrina. Para él, por ejemplo, las instituciones culturales presentan dos mecanismos de limitación de la variabilidad de las estructuras sociales: por una parte, los imperativos universales, que implican que las pautas orientadas de valor deben adaptarse a "ciertas exigencias motivacionales y de la situación que 
son universales a todas las especies humanas" (Parsons, 1951, p. 177); y, por otra, los imperativos estructurales, que suponen la compatibilidad de las instituciones con el sistema social en su conjunto. Con respecto a lo segundo afirma, por ejemplo, que la relación entre la moderna estructura ocupacional industrial y la familia solo permite que la última tome la forma de unidad conyugal que se aísla en un alto grado- de otras solidaridades. Es decir, "es posible tener, o bien este último tipo de estructura familiar, o bien una economía altamente industrializada, pero no ambas en la misma sociedad" (Parsons, 1951, p. 178). Así, Parsons limita la variabilidad de las estructuras sociales y, con ello, la posibilidad de conflicto institucional.

En la misma línea de argumentación, y separándose parcialmente de la doctrina original expuesta por Keynes, debemos comprender las aportaciones que, basadas en los trabajos previos desarrollados por Young (1928) y Myrdal (1944), realiza Kaldor $(1966 ; 1970)$ al debate institucional al hacer suyo el "principio de causación circular y acumulativa". Según el autor, las divergentes pautas de crecimiento económico no pueden ser analizadas como si no mantuvieran fuertes relaciones de interdependencia y retroalimentación entre sí, así como con los diferentes factores institucionales. De tal modo, Kaldor afirma que:

\begin{abstract}
Debido a los rendimientos crecientes en la fabricación, el éxito genera más éxito y el fracaso más fracaso (,,) Como resultado de ello, el libre comercio en el campo de los bienes manufacturados conduce a una concentración de la producción manufacturera en ciertas áreas; a un "proceso de polarización" que, al tiempo que inhibe el crecimiento de tales actividades en determinadas zonas, lo concentra en otras (Kaldor, 1996, p. 64, cursiva en el original).
\end{abstract}

Así, según Skott y Auerbach (1995), a fin de interpretar adecuadamente el concepto de causación acumulativa (según podemos encontrarlo en autores tales como Marx, Myrdal y Kaldor) hemos de atender a tres características. En primer lugar, dicha causación acumulativa remite siempre a la tradición, y "la existencia de mecanismos de retroalimentación desestabilizadores implica que las perturbaciones temporales pueden tener efectos grandes y acumulativos" (Skott y Auerbach, 1995, p. 382). En segundo lugar, "la inestabilidad, a menudo, surge de la interacción entre factores económicos y no económicos" (Skott y Auerbach, 1995, p. 382). Y, finalmente, "los conjuntos de 'posibilidades' relevantes vienen restringidos por la organización social e institucional de la actividad económica, así como por factores exógenos" (Skott y Auerbach, 1995, p. 383). Como podemos comprobar, esta forma de comprender la causación acumulativa, y muy especialmente la tercera característica, aproxima la doctrina de Kaldor a la parsoniana por cuanto el posible conflicto institucional se ve sensiblemente rebajado al reducirse la diversidad de formas institucionales posibles (aunque puedan persistir situaciones de desigualdad debido a la primera y segunda características).

\section{Conclusiones}

A la vista de los resultados (Tabla l), concluimos que, si bien Parsons comparte la caracterización de los agentes individuales que llevan a cabo tanto Keynes como los autores postkeynesianos, también se vale de modelos formales, como hacen los neokeynesianos, para dar cuenta de ellos. Además, se aproxima al estudio de las organizaciones atendiendo a dos áreas. Así, mientras que en la esfera intraorganizativa analiza la división del trabajo y el proceso de separación entre los ámbitos de la propiedad y de la gestión empresarial; en la interorganizativa legitima el papel que puede jugar el Estado en la economía y destaca, como los postkeynesianos, el positivo papel que pueden desarrollar los sindicatos. Finalmente, Parsons también coincide con los economistas postkeynesianos, y muy particularmente con Kaldor, por cuanto comprende las instituciones como fenómenos generales y naturales que pueden presentar un bajo perfil de conflictividad. 
Tabla 1. Contenido de las categorías analizadas en este artículo y ordenadas por autor y corriente de pensamiento

\begin{tabular}{|c|c|c|c|c|c|}
\hline \multicolumn{3}{|c|}{ Categorías } & \multirow{2}{*}{$\begin{array}{c}\begin{array}{c}\text { Keynes y } \\
\text { Postkeynesianos }\end{array} \\
\begin{array}{c}\text { Racionalidad } \\
\text { "material" }\end{array}\end{array}$} & \multirow{2}{*}{$\begin{array}{c}\text { Parsons } \\
\text { Modelo formal } \\
\text { (sistema de } \\
\text { personalidad) }\end{array}$} & \multirow{2}{*}{$\begin{array}{l}\text { Neokeynesianos } \\
\text { Modelo formal }\end{array}$} \\
\hline \multirow[t]{4}{*}{$\begin{array}{c}\text { Agente } \\
\text { individual }\end{array}$} & \multicolumn{2}{|c|}{ Modelo } & & & \\
\hline & \multicolumn{2}{|c|}{ Carácter maximizador } & \multicolumn{3}{|c|}{ Aceptación } \\
\hline & \multicolumn{2}{|c|}{$\begin{array}{c}\text { Capacidad de cálculo y disponibilidad } \\
\text { de información }\end{array}$} & \multicolumn{2}{|c|}{ Limitada } & Absoluta \\
\hline & \multicolumn{2}{|c|}{ Componente no racional } & \multicolumn{2}{|c|}{ Aceptación } & Rechazo \\
\hline \multirow[t]{5}{*}{$\begin{array}{l}\text { Organi- } \\
\text { zaciones }\end{array}$} & \multirow[t]{2}{*}{$\begin{array}{c}\text { Esfera } \\
\text { intraorganizativa }\end{array}$} & $\begin{array}{l}\text { División del } \\
\text { trabajo }\end{array}$ & \multirow{2}{*}{\multicolumn{3}{|c|}{ Aceptación }} \\
\hline & & $\begin{array}{l}\text { Separación } \\
\text { propiedad y } \\
\text { gestión }\end{array}$ & & & \\
\hline & \multirow{3}{*}{$\begin{array}{c}\text { Esfera } \\
\text { interorganizativa }\end{array}$} & Papel del Estado & \multicolumn{3}{|c|}{ Aceptación } \\
\hline & & Sindicatos & $\begin{array}{l}\text { Generan fricciones } \\
\text { que pueden } \\
\text { favorecer un } \\
\text { aumento de la } \\
\text { demanda agregada }\end{array}$ & $\begin{array}{l}\text { Desarrollan } \\
\text { funciones } \\
\text { semi-rituales que } \\
\text { favorecen la } \\
\text { autoestima }\end{array}$ & $\begin{array}{l}\text { Generan fricciones } \\
\text { y desempleo }\end{array}$ \\
\hline & & Competencia & \multicolumn{3}{|c|}{ Aceptación } \\
\hline \multirow[t]{4}{*}{ Instituciones } & \multicolumn{2}{|c|}{ Carácter general } & \multicolumn{2}{|c|}{ Aceptación } & \multirow{4}{*}{$\begin{array}{l}\text { No atienden a esta } \\
\text { categoría al basarse } \\
\text { en modelos de } \\
\text { sistemas } \\
\text { económicos } \\
\text { cerrados }\end{array}$} \\
\hline & \multicolumn{2}{|c|}{ Carácter natural } & \multicolumn{2}{|c|}{ Aceptación } & \\
\hline & \multicolumn{2}{|c|}{ Carácter espontáneo } & No atienden & Aceptación & \\
\hline & \multicolumn{2}{|c|}{ Carácter conflictivo } & $\begin{array}{c}\text { Keynes: aceptación. } \\
\text { Kaldor: rechazo } \\
\text { parcial }\end{array}$ & Rechazo parcial & \\
\hline
\end{tabular}

\section{Referencias bibliográficas}

Coddington, A. (1976). Keynesian economics: The search for first principles. Journal of Economic Literature, 14(4), 1258-1273.

Davidson, P. (1982). International money and the real world. Londres, Reino Unido: Palgrave Macmillan. https://doi.org/10.1007/978-1-349-16679-4

Garfinkel, H. (1968). Estudios en etnometodología. Barcelona/España, Ciudad de México/México y Bogotá/Colombia: Anthropos Editorial.

Habermas, J. (1987). La teoría de la acción comunicativa II. Crítica de la razón funcionalista. Madrid, España: Taurus Ediciones. 
Hernández, S. (2016). La fundamental convergencia teórica entre el pensamiento económico keynesiano y el estructural-funcionalismo sociológico de Talcott Parsons. Revista de Economía Crítica, 22(2), 49-64.

Joas, H. (1992). La creatividad de la acción. Madrid, España: CIS Centro de Investigaciones Sociológicas.

Joas, H. y Knöbl, W. (2004). Teoría social. Veinte lecciones introductorias. Madrid, España: Akal.

Kaldor, N. (1939). Speculation and economic stability. The Review of Economic Studies, 7(1), 1-27. https://doi.org/10.2307/2967593

Kaldor, N. (1966). Causes of the slow rate of economic growth of the United Kingdom: An inaugural lecture. Cambridge, UK: Cambridge University Press.

Kaldor, N. (1970). The case for regional policies. Scottish Journal of Political Economy, 17(3), 337-348. https://doi.org/10.1111/j.1467-9485.1970.tboo712.x

Kaldor, N. (1996). Causes of growth and stagnation in the world economy. Cambridge, Reino Unido: Cambridge University Press. https://doi.org/10.1017/cbo9780511559709

Keynes J. M. (1921). A treatise on probability. Londres, Reino Unido: Macmillan and Co., Limited.

Keynes, J. M. (1930). A treatise on money (II Vols.). Londres, Reino Unido: Macmillan and Co., Limited.

Keynes, J. M. (1936). Teoría general de la ocupación, el interés y el dinero. Ciudad de México/México y Buenos Aires/Argentina: Fondo de Cultura Económica.

Keynes, J. M. (1937). The general theory of employment. Quarterly Journal of Economics, 51(2), 209-223. https://doi.org/10.2307/1882087

Lamo de Espinosa, E. (2001). La sociología del siglo XX. REIS. Revista Española de Investigaciones Sociológicas, (96), 21-49. https://doi.org/10.2307/40184382

Lawson, T. (1994). The nature of post keynesianism and its links to other traditions: A realist perspective. Journal of Post Keynesian Economics, 16(4), 503-538. https://doi.org/10.1080/01603477. 1994.11489998

Leijonhufvud, A. (1968). On keynesian economics and the economics of Keynes: A study in monetary theory. Nueva York, Estados Unidos: Oxford University Press.

Merton, R. K. (1948). The seIf-fulfilling prophecy. The Antioch Review, 8(2), 193-210. https://doi. org/10.2307/4609267

Myrdal, G. (1944). An american dilemma: The negro problem and modern democracy (II Vols.). Nueva York, Estados Unidos: Harper \& Bros.

Parsons, T. (1937). La estructura de la acción social (II Vols.). Madrid, España: Ediciones Guadarrama.

Parsons, T. (1951). The social system. Nueva York, Estados Unidos: The Free Press of Glencoe.

Parsons, T. (1956a). Suggestions for a sociological approach to the theory of organizations I. Administrative Science Quarterly, 1(1), 63-85. https://doi.org/10.2307/2390840

Parsons, T. (1956b). Suggestions for a sociological approach to the theory of organizations II. Administrative Science Quarterly, 1(2), 225-239. https://doi.org/10.2307/2390988 
Parsons, T. (1970). Social structure and personality. Nueva York, Estados Unidos: The Free Press.

Parsons, T. (1971). The system of modern societies. Nueva Jersey, Estados Unidos: Prentice-Hall, Inc.

Parsons, T. (1974). Review of Harold J. Bershady, Ideology and social knowledge. Sociological Inquiry, 44(3), 215-221. https://doi.org/10.1111/j.1475-682x.1974.tboo742.x

Parsons, T. y Shils, E. A. (1951). Toward a general theory of action. Cambridge, Estados Unidos: Harvard. https://doi.org/10.4159/harvard.9780674863507

Parsons, T. y Smelser, N. J. (1956). Economy and society. Londres, Reino Unido: Routledge \& Kegan Paul.

Parsons, T., Bales, R. F. y Shils, E. A. (1953). Working papers in the theory of action. Glencoe, Estados Unidos: Free Press.

Robbins, L. (1932). An essay on the nature and significance of economic science. Londres, Reino Unido: Macmillan and Co.

Robinson, J. (1933). The economics of imperfect competition. Londres, Reino Unido: Macmillan and Co.

Robinson, J. (1937). Essays in the theory of employment. Londres, Reino Unido: Macmillan and Co.

Robinson, J. (1953-1954). The production function and the theory of capital. The Review of Economic Studies, 21(2), 81-106. https://doi.org/10.2307/2296002

Robinson, J. (1980). Time in economic theory.Kyklos, 3(2), 219-229. https://doi.org/10.1111/j.1467-6435.1980. tbo2632.x

Samuelson, P. A. (1938). A note on the pure theory of consumers' behaviour. Economica, 5(17), 61-71. https://doi.org/10.2307/2548836

Samuelson, P. A. (1952). Economic theory and mathematics: An appraisal. The American Economic Review, 42(2), 56-66.

Samuelson, P. A. (1969). Classical and neoclassical theory. En R. W. Clower (Ed.), Monetary theory (pp. 51-73). Londres, Reino Unido: Penguin Books.

Samuelson, P. A. (1970). Curso de economía moderna. Una descripción analítica de la realidad económica. Madrid, España: Aguilar.

Samuelson, P. A. (1983). Foundations of economic analysis (Enlarged ed.). Cambridge/Estados Unidos y Londres/Reino Unido: Harvard University Press.

Samuelson, P. A. y Nordhaus, W. D. (2005). Economía (18 ed.). Ciudad de México, México: McGraw Hill Interamericana.

Searle, J. R. (1995). La construcción de la realidad social. Barcelona, España: Paidós.

Shackle, G. L. S. (1955). Uncertainty in economics and other reflections. Cambridge, Reino Unido: Cambridge University Press.

Shackle, G. L. S. (1961). Decision, order and time in human affairs. Cambridge, Reino Unido: Cambridge University Press. 
Shackle, G. L. S. (1967). The years of high theory: Invention and tradition in economic thought 1926-1939. Cambridge, Reino Unido: Cambridge University Press.

Skott, P. y Auerbach, P. (1995). Cumulative causation and the "new" theories of economic growth. Journal of Post Keynesian Economics, 17(3), 381-402. https://doi.org/10.1080/01603477.1995.11490036

Young, A. (1928). Increasing returns and economic progress. Economic Journal, 38(152), 527-542. https:// doi.org/10.2307/2224097 